\title{
EMBEDDING INVERSE SEMIGROUPS IN WREATH PRODUCTS
}

\author{
by C. H. HOUGHTON
}

(Received 21 August, 1974)

1. Introduction. Any extension of a group $A$ by a group $B$ can be embedded in their wreath product $A \mathrm{Wr} B$. Here we consider generalizations of this result for inverse semigroups.

Suppose $S$ is an inverse semigroup and $\rho_{0}$ is a congruence on $S$. We put $T=S / \rho_{0}$ and denote the natural map from $S$ to $T$ by $\rho$. The kernel of $\rho$ is the inverse image $E_{T} \rho^{-1}$ of the semilattice $E_{T}$ of idempotents of $T$. First we show that if each $\rho_{0}$-class of idempotents of $S$ is inversely well-ordered, then $S$ can be embedded in $K \mathrm{Wr} T$, the standard wreath product of $K$ and $T$. In general, not all elements of $K \mathrm{Wr} T$ have inverses. However, we can define a wreath product $W(K, T)$ which is an inverse semigroup and which contains $S$ when the previous condition holds. If $\rho_{0}$ is idempotent-separating and $S$ is 0 -bisimple, $K$ is the union of zero and a family of isomorphic groups. In this case, we can replace $K$ by a single component group $G$ of $K$, augmented by zero, and show that $S$ can be embedded in $W\left(G^{0}, T\right)$. These results are analogous to the extension theories of D'Alarcao [1] and Munn [3] and they give conditions under which all inverse semigroup extensions of an inverse semigroup $A$ by an inverse semigroup $T$ are contained in a semigroup with structure depending only on $A$ and $T$.

N. R. Reilly (see [6]) has previously obtained results related to some of those presented here, using a construction generalizing the wreath product of permutation groups. I thank the referee for his comments and for informing me of Reilly's work.

2. Embedding in the standard wreath product. We recall the definition of the standard wreath product of two semigroups, due to Neumann [4].

Let $A$ and $T$ be inverse semigroups and let $F$ denote the set of maps from $T$ to $A$. Then $F$ is an inverse semigroup with respect to multiplication given by $x(f g)=(x f)(x g)$ for $x \in T$ and $f, g \in F$. For $f \in F$ and $t \in T$, we define $' f \in F$ by $x\left({ }^{t} f\right)=(x t) f$ for all $x \in T$. The wreath product $W=A$ Wr $T$ is the semigroup consisting of all pairs $f t$ with $f \in F, t \in T$, under the multiplication $(f t)(g u)=\left(f .^{t} g\right)(t u)$, for $f, g \in F, t, u \in T$. In general, $W$ is not an inverse semigroup; if $A$ and $T$ contain zero, the element $f t$ has no inverse if we take $t=0,0 f=0$ and $x f \neq 0$, for some $x \in T$.

Given an inverse semigroup $S$ and a congruence $\rho_{0}$ on $S$ such that $T=S / \rho_{0}$, we consider the possibility of constructing a homomorphism $\omega$ from $S$ to $A$ Wr $T$ such that $s \omega=f_{s}(s \rho)$, where $\rho$ is the natural map from $S$ to $T$ and $f_{s} \in F$. Clearly this is equivalent to finding $\left\{f_{s}: s \in S\right\} \subseteq F$ with $f_{r s}=f_{r} \cdot{ }^{r \rho} f_{s}$ for all $r, s \in S$. Evaluating this expression gives

$$
t f_{r s}=\left(t f_{r}\right)\left((t . r \rho) f_{s}\right)
$$

for all $t \in T$. If we require $\omega$ to be injective, the corresponding condition on $\left\{f_{s}\right\}$ is that if $r \rho=s \rho$ but $r \neq s$, then there exists $t \in T$ such that $t f_{r} \neq t f_{s}$.

Let us define a partial multiplication on the set $T \times S$ by putting $(t, r)(u, s)=(t, r s)$ 
whenever $t(r \rho)=u$. Then $T \times S$ is an associative partial groupoid, in the sense of Clifford and Preston [2].

Lемма 1. Let $\pi: W \rightarrow T$ be the projection map. There is a one-one correspondence between the homomorphisms $\omega$ from $S$ to $W$ such that $\omega \pi=\rho$ and the homomorphisms $\beta$ from $T \times S$ to $A$. Furthermore, $\omega$ is injective if and only if the corresponding $\beta$ has the property that for $r, s \in S$ with $r \rho=s \rho$ but $r \neq s$, there exists $t \in T$ such that $(t, r) \beta \neq(t, s) \beta$.

Proof. Given such an $\omega$, with $s \omega=f_{s}(s \rho)$, we put $(t, s) \beta=t f_{s^{*}}$ Conversely, given $\beta$, put $s \omega=f_{\mathrm{s}}(s \rho)$, with $t f_{s}=(t, s) \beta$. The results follow immediately.

We shall now give sufficient conditions for the existence of a homomorphism $\beta$ from $T \times S$ to the kernel $K$ of $\rho$.

Let $E_{S}$ be the set of idempotents of $S$ and recall that $E_{S}$ is partially ordered by the relation $e \leqq f$ if and only if $e f=e$. Suppose $\tau$ is a left inverse of $\rho$ such that, for all $t \in T$ and $s \in S$,

$$
(t \tau . s)^{-1} t \tau . s \leqq((t . s \rho) \tau)^{-1}(t . s \rho) \tau .
$$

For $(t, s) \in T \times S$, put

$$
(t, s) \gamma=\left(t t^{-1}, t \tau . s((t . s \rho) \tau)^{-1}\right)
$$

We show that $\gamma$ is a homomorphism from $T \times S$ to its subgroupoid $M$ consisting of those $(e, k)$ with $e, k \rho \in E_{S}$ and $k \rho \leqq e$. If $r \in S$ then

$$
\begin{aligned}
(t, s) \gamma .(t . s \rho, r) \gamma & =\left(t t^{-1}, t \tau . s((t . s \rho) \tau)^{-1}\right)\left(t . s \rho(t . s \rho)^{-1},(t . s \rho) \tau . r((t . s \rho . r \rho) \tau)^{-1}\right) \\
& =\left(t t^{-1}, t \tau . s((t . s \rho) \tau)^{-1}(t . s \rho) \tau . r((t .(s r) \rho) \tau)^{-1}\right) .
\end{aligned}
$$

By the assumption on $\tau$,

$$
t \tau . s((t . s \rho) \tau)^{-1}(t . s \rho) \tau=t \tau . s
$$

and so

$$
(t, s) \gamma \cdot(t \cdot s \rho, r) \gamma=(t, s r) \gamma .
$$

We note that if $(t, s) \gamma=(e, k)$ then

$$
k \rho=t . s \rho(t . s \rho)^{-1}=t(s \rho)(s \rho)^{-1} t^{-1} \leqq t t^{-1}=e .
$$

If $(e, k),(k \rho, h) \in M$ then $(e, k)(k \rho, h)=(e, k h)$. Thus the projection map $\delta$, given by $(e, k) \delta=k$, is a homomorphism from $M$ to the kernel $K$ of $\rho$. Then $\beta=\gamma \delta$ is a homomorphism from $T \times S$ to $K$.

Suppose now that for each $s \in S$ there exists $t \in T$ such that $(t \tau)^{-1} t \tau \geqq s s^{-1}$. For such $t$ and $s$, using the previous assumption on $\tau$,

$$
((t . s \rho) \tau)^{-1}(t . s \rho) \tau \geqq(t \tau . s)^{-1} t \tau . s=s^{-1}(t \tau)^{-1} t \tau . s=s^{-1} s .
$$

Now $(t, s) \beta=t \tau . s((t . s \rho) \tau)^{-1}$ and so

$$
(t \tau)^{-1}(t, s) \beta .(t . s \rho) \tau=(t \tau)^{-1} t \tau . s .(t . s \rho) \tau((t . s \rho) \tau)^{-1}=s .
$$

Thus if $r \rho=s \rho$ but $r \neq s$, then $(t, r) \beta \neq(t, s) \beta$.

We summarise and apply these results. 
Lemma 2. Suppose $\rho$ has a left inverse $\tau$ such that

(i) for all $t \in T$ and $s \in S$,

$$
(t \tau . s)^{-1} t \tau . s \leqq((t . s \rho) \tau)^{-1}(t . s \rho) \tau,
$$

(ii) for each $s \in S$ there exists $t \in T$ such that $(t \tau)^{-1} t \tau \geqq s s^{-1}$.

Then $\beta$, given by

$$
(t, s) \beta=t \tau . s((t . s \rho) \tau)^{-1},
$$

is a homomorphism from $T \times S$ to $K=\operatorname{Ker} \rho$, satisfying the conditions of Lemma 1 with $A=K$.

We recall that a partially ordered set is inversely well-ordered if each subset has a greatest element.

THeOREM 3. Let $S$ be an inverse semigroup and let $\rho$ be a homomorphism from $S$ onto $T$ such that, for each $e \in E_{T}, e \rho^{-1} \cap E_{S}$ is inversely well-ordered. Then there is a monomorphism $\omega$ from $S$ to $K \mathrm{Wr} T$ such that $\omega \pi=\rho$, where $K$ is the kernel of $\rho$ and $\pi$ is the projection map from $K \mathrm{Wr} T$ to $T$.

Proof. We find a map $\tau$ satisfying the conditions of Lemma 2. For $t \in T$ and $s \in t \rho^{-1}$ we have $s^{-1} s \in\left(t^{-1} t\right) \rho^{-1} \cap E_{S}$. Choose $s \in t \rho^{-1}$ such that $s^{-1} s$ is maximal in $\left(t^{-1} t\right) \rho^{-1} \cap E_{S}$ and define $\tau$ by the rule that $t \tau=s$. For any $t \in T, s \in S$, we have $(t \tau . s) \rho=t . s \rho$ so

$$
(t \tau . s)^{-1} t \tau . s \leqq((t . s \rho) \tau)^{-1}(t . s \rho) \tau .
$$

Given $u \in T$, for $s \in u \rho^{-1}$ it follows that $\left(s s^{-1}\right) \rho=u u^{-1}$ and

$$
\left(\left(u u^{-1}\right) \tau\right)^{-1}\left(u u^{-1}\right) \tau \geqq\left(s s^{-1}\right)^{-1} s s^{-1}=s s^{-1} .
$$

From Lemmas 2 and $1, \omega$, given by $s \omega=f_{s}(s \rho)$ with $t f_{s}=t \tau . s((t . s \rho) \tau)^{-1}$, is a monomorphism of the required type.

We note that the result of Theorem 3 holds for any idempotent-separating homomorphism of an arbitrary inverse semigroup. On the other hand, it holds for an arbitrary homomorphism of an inverse semigroup with inversely well-ordered semilattice of idempotents.

3. Embedding in a wreath product related to principal left ideals. We now define a wreath product of inverse semigroups which is itself an inverse semigroup and for which the embedding result of Theorem 3 holds. Let $A$ and $T$ be inverse semigroups and let $F$ now consist of all maps from principal left ideals of $T$ to $A$. We denote the domain of $f \in F$ by $D(f)$ and recall that each principal left ideal has an idempotent generator, since $T t=T t^{-1} t$. Thus if $f, g \in F$ and $D(f)=T d, D(g)=T e$, with $d$, e $\in E_{T}$, then $D(f) \cap D(g)=T d e$. We define $f g$ by taking $D(f g)=D(f) \cap D(g)$ and $x(f g)=(x f)(x g)$ for $x \in D(f g)$. Then $F$ is an inverse semigroup.

Suppose $t \in T$ and $f \in F$. Since $D(f)=T e$, for some $e \in E_{T}$, we have

$$
D(f) t^{-1} t=T e t^{-1} t \subseteq T e=D(f) .
$$

Putting $x\left({ }^{t} f\right)=(x t) f$ for all $x \in D(f) t^{-1}$, we see that ${ }^{t} f \in F$ with $D\left({ }^{t} f\right)=D(f) t^{-1}$. We now define $W=W(A, T)$ to consist of all pairs $f t$ with $f \in F, t \in T$ and $D(f)=T t^{-1}$. If $e=t t^{-1}$, 
the last condition implies that $x e=x$ for $x \in D(f)$ and hence ${ }^{c} f=f$. As before, the rule $(f t)(g u)=\left(f \cdot{ }^{t} g\right)(t u)$ defines an associative multiplication on $W$; we note that

$$
D\left(f .^{t} g\right)=D(f) \cap D(g) t^{-1}=T t^{-1} \cap T u^{-1} t^{-1}=T(t u)^{-1} .
$$

If $g u \in W$ is an inverse of $f t \in W$ then

and

$$
g u=g u \cdot f t \cdot g u=(g \cdot " f \cdot " g)(u t u)
$$

$$
f t=f t \cdot g u \cdot f t=\left(f \cdot{ }^{\prime} g \cdot{ }^{t u} f\right)(t u t) \text {. }
$$

Thus $u=t^{-1}$ and so ${ }^{t t} g=g,{ }^{t u} f=f$. Then $g=g \cdot{ }^{u} f . g$ and $" f=" f . g . " f$, so $g=(" f)^{-1}$. Conversely, if $u=t^{-1}$, then $\left({ }^{u} f\right)^{-1} u \in W$ and is an inverse of $f t$. Thus $W$ is an inverse semigroup. We call $W=W(A, T)$ the wreath product of $A$ and $T$ with respect to principal left ideals. It is related to the "partial wreath product" defined by Petrich [5].

As before, we suppose $\rho$ is a homomorphism from $S$ onto $T$ and we consider the possibility of constructing a homomorphism $\omega$ from $S$ to $W$ such that $s \omega=f_{s}(s \rho)$. The argument follows the previous lines, except that we now have to consider the domains of the functions involved.

Let $C$ be the subset of $T \times S$ consisting of those $(t, s)$ such that $t \in T(s \rho)^{-1}$. If $(t, s)$, $(t . s \rho, r) \in C$, then $t . s \rho \in T(r \rho)^{-1}$ and so $t=t(s \rho)(s \rho)^{-1} \in T((s r) \rho)^{-1}$ and $(t, s r) \in C$. Thus $C$ is a subgroupoid of the associative partial groupoid $T \times S$. Suppose $\theta$ is a homomorphism from $C$ to an inverse semigroup $A$. Define a map $\omega: S \rightarrow W=W(A, T)$ by the rule that $s \omega=f_{s}(s \rho)$, where $f_{s}$ is the map from $T(s \rho)^{-1}$ to $A$ given by $t f_{s}=(t, s) \theta$. Then $\omega$ will be a homomorphism if and only if $f_{r s}=f_{r} \cdot{ }^{r \rho} f_{s}$ for all $r, s \in S$. Now

$$
\begin{aligned}
D\left(f_{r} \cdot{ }^{r \rho} f_{s}\right) & =D\left(f_{r}\right) \cap D\left({ }^{r \rho} f_{s}\right)=T(r \rho)^{-1} \cap T(s \rho)^{-1}(r \rho)^{-1} \\
& =T((r s) \rho)^{-1}=D\left(f_{r s}\right)
\end{aligned}
$$

and, for $t \in D\left(f_{r s}\right)$,

$$
t\left(f_{r} \cdot{ }^{r \rho} f_{s}\right)=(t, r) \theta \cdot(t \cdot r \rho, s) \theta=(t, r s) \theta=t f_{r s}
$$

as required.

Suppose that $e \rho^{-1} \cap E_{S}$ is inversely well-ordered, for all $e \in E_{T}$. In the proof of Theorem 3, we constructed a map $\tau$ satisfying the conditions of Lemma 2 and thus giving rise to homomorphisms $\beta$ and $\gamma$ from $T \times S$ to $K$ and $E_{T} \times K$ respectively, defined by

$$
(t, s) \beta=t \tau . s((t . s \rho) \tau)^{-1}
$$

and $(t, s) \gamma=\left(t t^{-1},(t, s) \beta\right)$.

THEOREM 4. Let $S$ be an inverse semigroup and let $\rho$ be a homomorphism from $S$ onto $T$ such that, for each $e \in E_{T}, e \rho^{-1} \cap E_{S}$ is inversely well-ordered. Then there is a monomorphism $\omega$ from $S$ to $W=W(K, T)$ such that $\omega \pi=\rho$, where $K$ is the kernel of $\rho, W$ is the wreath product of $K$ and $T$ with respect to principal left ideals, and $\pi$ is the projection map from $W$ to $T$.

Proof. From the remarks above, the restriction $\theta$ of $\beta$ to $C$ corresponds to a homomorphism $\omega$ from $S$ to $W$ with $\omega \pi=\rho$, and it remains to prove that $\omega$ is injective. If $r, s \in S$ with $r \omega=s \omega$ then $r \rho=s \rho$ and $f_{r}=f_{s}$. In particular, since $e=\left(s s^{-1}\right) \rho \in T(s \rho)^{-1}=D\left(f_{s}\right)$, we have $e f_{r}=e f_{s}$ and so $(e, r) \beta=(e, s) \beta$. For $t \in T, t \tau$ was chosen so that $(t \tau)^{-1}(t \tau)$ is maximal in 
$\left(t^{-1} t\right) \rho^{-1} \cap E_{s}$. Taking $t=e=\left(s s^{-1}\right) \rho$, we have $(e \tau)^{-1}(e \tau) \geqq s s^{-1}$, and taking $t=s \rho$, we have $(s \rho \tau)^{-1}(s \rho \tau) \geqq s^{-1} s$. Then

$$
\begin{aligned}
s & =s s^{-1} . s . s^{-1} s=(e \tau)^{-1}(e \tau) s s^{-1} . s . s^{-1} s(s \rho \tau)^{-1}(s \rho \tau) \\
& =(e \tau)^{-1}(e \tau) s(s \rho \tau)^{-1}(s \rho \tau)=(e \tau)^{-1} .(e, s) \beta .(s \rho \tau) .
\end{aligned}
$$

Since $r \rho=s \rho$, a similar argument shows that $r=(e \tau)^{-1} .(e, r) \beta .(r \rho \tau)$ and hence $r=s$.

Finally we consider the case where $S$ is 0 -bisimple and $\rho$ is idempotent-separating. In this situation, the $\mathscr{H}$-class of an idempotent $e$ of $S$ contains $(e \rho) \rho^{-1}$ as a subgroup. Further more, the isomorphism of [2, Theorem 2.20] between the $\mathscr{H}$-classes of two non-zero idempotents of $S$ induces an isomorphism between the corresponding subgroups. Thus the inverse images of non-zero idempotents of $T$ are isomorphic groups. For $G$ representing such a group, we shall construct a homomorphism from $C$ to $G^{0}$ which leads to an embedding of $S$ in $W=W\left(G^{0}, T\right)$.

Since $\rho$ is idempotent-separating, $e \rho^{-1} \cap E_{S}$ is trivially inversely well-ordered, for $e \in E_{T}$, and so we are in the situation of Theorem 4. Then $\gamma$, defined by $(t, s) \gamma=\left(t t^{-1},(t, s) \beta\right)$, induces a homomorphism from $C$ to $E_{T} \times K$. For $(t, s) \in C$, we have $t \in T(s \rho)^{-1}$ and so

$$
(t, s) \beta \rho=\left(t \tau . s((t . s \rho) \tau)^{-1}\right) \rho=t(s \rho)(s \rho)^{-1} t^{-1}=t t^{-1} .
$$

Thus the image of $C$ under $\gamma$ is contained in the set $N$ of all $(e, k)$ with $k \in e \rho^{-1}, e \in E_{T}$. Now $N$ is the disjoint union of a zero element $(0,0)$ and copies of $G$, with multiplication defined only in the components of the union. Thus there is a homomorphism $\alpha$ from $N$ to $G^{0}$ inducing a monomorphism on each component of $N$. Combining $\gamma$ and $\alpha$ we have a homomorphism $\phi=\gamma \alpha$ from $C$ to $G^{0}$. The map $\omega$ from $S$ to $W\left(G^{0}, 7\right)$ corresponding to $\phi$ will be a homomorphism and it remains to show that $\omega$ is injective.

If $r, s \in S$ with $r \omega=s \omega$ then $r \rho=s \rho$ and $f_{r}=f_{s}$; in particular, $e f_{r}=e f_{s}$, for $e=\left(s s^{-1}\right) \rho$. Then

$$
(e,(e, r) \beta) \alpha=(e, r) \phi=e f_{r}=e f_{s}=(e, s) \phi=(e,(e, s) \beta) \alpha .
$$

Now $\alpha$ acts as a monomorphism on the component groups of $N$, and $(e,(e, r) \beta),(e,(e, s) \beta)$ are in the same component. Thus $(e, r) \beta=(e, s) \beta$ and it follows from the proof of Theorem 4 that $r=s$. We have thus proved the following result.

THEOREM 5. Let $S$ be a 0-bisimple inverse semigroup and let $\rho$ be an idempotent-separating homomorphism from $S$ onto $T$. Let $G$ be a group isomorphic to the inverse images of the non-zero idempotents of $T$. Then there is a monomorphism $\omega$ from $S$ to $W$ such that $\omega \pi=\rho$, where $W=W\left(G^{0}, T\right)$ is the wreath product of $G^{0}$ and $T$ with respect to principal left ideals and $\pi$ is the projection map from $W$ to $T$.

\section{REFERENCES}

1. H. D'Alarcao, Idempotent-separating extensions of inverse semigroups, J. Austral. Math. Soc. 9 (1969), 211-217. 1961).

2. A. H. Clifford and G. B. Preston, The algebraic theory of semigroups, Vol. I (Providence, R.I.,

3. W. D. Munn, 0-bisimple inverse semigroups, J. Algebra 15 (1970), 508-85. 
4. B. H. Neumann, Embedding theorems for semigroups, J. London Math. Soc. 35 (1960), 184-192.

5. M. Petrich, Introduction to semigroups (Columbus, Ohio, 1973).

6. N. R. Reilly, Enlarging the Munn representation, submitted to J. Austral. Math. Soc.

UNIVERSITY COLlege

CARDIFF 